\title{
DA MOVIMENTO ERETICALE A CHIESA RIFORMATA: UNA LETTURA DEL VALDISMO ATTRAVERSO LE SUE CONFESSIONI DI FEDE
}

\author{
Andrea Arcuri \\ Universidad de Granada / Universidad de Palermo
}

Sommario: Con questo articolo si è inteso analizzare l'incontro tra il Valdismo e la Riforma, e la trasformazione del movimento ereticale medievale in chiesa riformata attraverso l'esame di due confessioni di fede: la Dichiarazione del Sinodo di Chanforan (1532), mediante la quale si sancisce l'adesione dei valdesi alla Riforma, e la Confessione di fede valdese del 1655, dove è possibile rintracciare i caratteri di una chiesa ormai ben definita, dotata di una dottrina, una organizzazione ed una disciplina ecclesiastica dal profilo marcatamente protestante.

Parole chiave: Valdismo, Confessioni di Fede, Età Moderna, Protestantesimo, Chiese Riformate.

\section{De movimiento herético a iglesia reformada: una lectura del valdismo mediante sus confesiones de fe}

Resumen: Este artículo tiene por objeto analizar el encuentro entre Valdismo y Reforma, y la trasformación del movimiento herético medieval en iglesia reformada a través del examen de dos confesiones de fe: la Declaración del Sínodo de Chanforan (1532), por medio de la cual se determina la incorporación de los valdenses a la Reforma, y la Confesión de fe valdense de 1655, en la que podemos relevar los rasgos de una iglesia ya claramente definida, provista de una doctrina, una organización y una disciplina eclesiástica marcadamente protestantes.

Palabras clave: Valdismo, Confesiones de Fe, Edad Moderna, Protestantismo, Iglesias Reformadas.

From heretical movement to reformed church: a view on waldism through its confessions of faith

Abstract: The aim of this article is to analyse the encounter between Waldism and Reformation, and the transformation of the medieval heretical movement into a reformed church through examination of two confessions of faith: Chanforan Synod Declaration (1532), that states the Waldensian adhesion to the Reform, and the Waldensian Confession of Faith of 1655 in which it is possible to trace an already well organized church with a doctrine and an ecclesiastical discipline prominently Protestant.

Key words: Waldism, Confessions of Faith, Modern Age, Protestantism, Reformed Churches.

Data de recepció: 30 d'octubre de 2017 / Data d'acceptació: 27 de novembre de 2017. 


\section{INTRODUZIONE}

La lunga storia del cristianesimo è stata contrassegnata da numerose richieste di renovatio, di riforma della struttura ecclesiastica e di una più stretta aderenza al messaggio evangelico. La richiesta di una reformatio della chiesa intesa come renovatio precede di secoli l'avvio della Riforma: "fin dal XIII secolo il cristianesimo occidentale era un magma ribollente di movimenti religiosi che anelavano a riportare la chiesa alla sua funzione puramente spirituale" (Campi, 2001, 6).

Il valdismo, movimento pauperistico ereticale, nato nel tardo XII secolo sull'esempio di Valdo di Lione, attraversò e sopravvisse a oltre tre secoli di clandestinità, di persecuzioni e di repressioni, prima di entrare in contatto con quell'ampio fenomeno di rinnovamento religioso rappresentato dalla Riforma (Merlo, 2010; Molnar, 1989, 9-34).

Il movimento conobbe comunque un periodo di espansione nel corso del XIII e del XIV secolo, determinato dal carattere missionario della sua predicazione che lo portò ad insediarsi dalla originaria Francia in Italia, dove già operavano i "Poveri lombardi", nell'area alpina e successivamente in Calabria e Puglia; altre aree in cui si insediarono comunità valdesi furono la Germania, i territori austriaci, la Polonia e la Boemia ${ }^{1}$.

L'area di maggiore penetrazione e radicamento del movimento valdese fu indubbiamente quella che comprendeva il Delfinato e le valli alpine tanto del versante francese che di quello italiano (Molnar, 1989, 75-120).

Il messaggio dei Poveri di Lione - altro modo con cui venivano indicati gli appartenenti al movimento - non si fondava su una teologia strutturata, presentava piuttosto caratteristiche e precetti che riguardavano la vita sociale e morale dei cristiani, interpretando il Vangelo come una lex Christi a cui uniformarsi: il nucleo della predicazione valdese, basato sul "Sermone del monte" [Mt. 5,1;7,29], si concentrava sulla semplicità della vita apostolica, il cui elemento sostanziale era rappresentato dalla predicazione itinerante in una condizione di povertà (Merlo, 1984; Cegna, 1981; Polastro, 1974).

Sul piano della concezione della chiesa, i valdesi, che si collocavano nel solco del cristianesimo primitivo, si opponevano al "costantinianesimo" che aveva dato forma alla chiesa romana, cui contestavano le ricchezze e il potere

${ }^{1}$ Qui il valdismo, nel XV secolo, si innestò nei fermenti politico-religiosi che agitavano la regione; secondo Amedeo Molnar, l'hussitismo, in particolare la fazione taborita, sarebbe stato fortemente influenzato dalla dottrina valdese (Molnar, 1986; Molnar, 1989, 159-96). 
mondano. Accettando la "donazione di Costantino" - che secondo la tradizione attribuiva alla chiesa il dominio temporale e la regalità imperiale - papa Silvestro, a giudizio dei seguaci di Valdo, aveva compiuto un atto di apostasia (Molnar, 1976; Gonnet, 1972): "per i valdesi l'accantonamento del mito di Costantino, la contestazione della teoria d'un papato erede dell'impero romano e di un impero ufficiato e consacrato per la sua missione religiosa, erano [...] una nozione teologica che permetteva di distinguere la vera dalla falsa chiesa" (Molnar, 1989, 309).

In ordine ai rapporti con le autorità secolari, professavano una separazione radicale, rifiutando il giuramento e l'uso della spada al servizio del potere ecclesiastico e civile. Anche per queste ragioni, che mettevano in discussione l'ordinamento stabilito e tendevano a desacralizzare il potere, il movimento venne duramente perseguitato (Molnar, 1989, 133-39).

Alla vigilia della Riforma la presenza valdese, che nel XIV secolo si estendeva dal sud dell'Italia fino alla Polonia, si era ridotta, per via di sanguinose persecuzioni, alle sole comunità della Provenza, del Delfinato, delle valli alpine occidentali, della Calabria e della Puglia, costrette a un'esistenza semiclandestina. Il timore delle repressioni obbligò i valdesi a pratiche nicodemitiche ${ }^{2}$, a "mimetizzarsi" nella società dell'epoca, partecipando alle celebrazioni della chiesa romana e ricevendo i sacramenti da parte di un clero cui non riconoscevano alcuna autorità; la "vera fede" veniva praticata in segreto (Subilia, 1982; Vinay, 1974; Comba, 1932).

Nelle prossime pagine - attraverso l'analisi di due confessioni di fede, la Dichiarazione del Sinodo di Chanforan (1532) e la Confessione di fede valdese del 1655 - si prende in esame il modo in cui l'incontro tra i valdesi e la Riforma protestante - con la sua spinta alla confessionalizzazione (Reinhard, 1977; Schilling, 1981) - abbia prodotto la trasformazione del valdismo medievale in una chiesa riformata, dotata di una organizzazione ecclesiastica definita.

\section{L'ADESIONE DEI VALDESI ALLA RIFORMA: LA DICHIARAZIONE DEL SINODO DI CHANFORAN (1532)}

Per un movimento di rinnovamento religioso come il valdismo, l'incontro con la Riforma rappresentò l'occasione di un confronto con un'ampia ed erudita platea di accademici, teologi e riformatori. I primi contatti con la Riforma

2 Il termine "nicodemismo" venne coniato da Calvino per indicare quanti non osavano professare pubblicamente la propria fede. Si riferiva al passo biblico [Gv. 3,2] in cui Nicodemo, per timore di essere scoperto dai Giudei, si reca di notte da Gesù (Campi, 1991, 179). 
si ebbero a partire dalla metà degli anni Venti del XVI secolo: il Sinodo di Laus (1526) decise di inviare in Svizzera due barba - termine con cui venivano indicati i predicatori itineranti valdesi (Audisio, 2007) - per conoscere i caratteri della nuova predicazione evangelica, che proprio in quegli anni cominciava ad affermarsi nelle città-stato elvetiche. L'interlocutore principale fu il riformatore Guglielmo Farel, la cui teologia apparve persuasiva ai due delegati, che al loro rientro nelle valli alpine vi diffusero la nuova letteratura riformata, accrescendovi l'interesse e l'entusiasmo per le idee religiose dei riformatori. Se i valdesi delle Alpi e della Provenza coglievano i benefici dell'incontro con la Riforma, anche i riformatori erano interessati ad attrarre quest' antica comunità religiosa, cui riconoscevano il titolo di Mater reformationis nelle fila del movimento evangelico che si andava sviluppando (Armand Hugon, 1989, 11).

L'esigenza di una esaustiva comprensione delle dottrine e dell'organizzazione ecclesiastica delle chiese della Riforma spinse i valdesi a condurre un'indagine più approfondita inviando, come stabilito nel Sinodo di Mérindol del 1530, i barba Georges Morel e Pierre Masson in Svizzera e nella Germania meridionale presso i riformatori. Nel corso della loro missione i barba si incontrarono e si confrontarono con Farel a Neuchâtel, con Ecolampadio a Basilea, con Haller a Berna e con Bucero a Strasburgo (Gonnet, 1981).

Per comprendere lo spirito che animava le comunità valdesi e le loro aspettative nei confronti della Riforma, è indicativa la corrispondenza che precede la missione dei due delegati; si tratta di lettere inviate ai riformatori dalle quali traspare il rispetto e l'ammirazione per la loro conoscenza delle Scritture e dell'interpretazione che ne danno. Un esempio di tale atteggiamento si può apprezzare nella lettera che i due barba inviarono ad Ecolampadio nell'ottobre del 1530: "Salve, mio benedetto signore Ecolampadio. Perché colui che può ogni cosa ha fatto giungere sempre più di frequente alla nostre orecchie, per mezzo di molti relatori, che egli ti ha benedetto col suo spirito, come dai frutti si può facilmente conoscere, siamo venuti a te da un paese lontano con l'animo pieno di esultanza. Speriamo e abbiamo piena fiducia che lo spirito menzionato per mezzo di te ci illumini e ci chiarisca molte cose, che per colpa della nostra ignoranza e pigrizia sono a noi dubbie e anche del tutto occulte, e ciò, come assai temo, con grave danno nostro e del popolo che ammaestriamo in modo tanto inadeguato. [...] Per la pigrizia della nostra natura non abbiamo in nessun modo inteso le Scritture tanto rettamente quanto voi. Perciò, siamo venuti a te per essere diretti, istruiti, edificati e ammaestrati" (I barba Georges Morel e Pierre Masson a Ecolampadio, 1530, 37 e 51).

Nei successivi incontri i barba esposero le dottrine, l'organizzazione e le tradizioni in uso nelle comunità valdesi: dalla persistenza del ministero itinerante al nicodemismo sacramentale indotto dalle necessità di una vita clandes- 
tina. Le risposte che ottennero andavano in una direzione differente dal carattere e dalle pratiche tradizionali del valdismo medievale: Farel, Ecolampadio e Bucero, con diverse sfumature, non mancarono di sottolineare l'opportunità di adottare diverse correzioni sia in ordine all'organizzazione ecclesiastica che sui temi della dottrina - giustificazione per fede, servo arbitrio, predestinazione, sacramenti - allineando, in tal modo, la teologia valdese con il pensiero della Riforma. Sul tema dei rapporti con l'autorità secolare i riformatori - in particolare Bucero, alle prese con la diffusione dell'anabattismo a Strasburgo esortarono i valdesi ad adottare un atteggiamento positivo nei confronti dei magistrati, in accordo con il senso dell'epistola di San Paolo ai Romani: "iudices et magistratus seculares [...] vicarii Dei sunt" e "Quae per magistratus fiunt, Dei sunt judicia" scrivono Ecolampadio e Bucero ai barba (Prima risposta di Ecolampadio ai valdesi, 1530, 58; Risposta di Martin Bucero ai fratelli Valdesi in Francia, 1530, 104).

La reazione rispetto al nicodemismo fu piuttosto dura, la Parola di Dio deve essere predicata pubblicamente; nella risposta di Ecolampadio ai valdesi emerge un giudizio molto severo al riguardo: "quelli che si saranno vergognati di confessare Cristo davanti al mondo, un giorno neppure dal padre suo saranno riconosciuti [Mt. 10,33]. Poiché l'Iddio nostro è verità, egli vuole che coloro che lo servono, lo servano in verità, senza alcun inganno di simulazione; egli è geloso e non tollera che i suoi portino il gioco con l'anticristo. [...] Però noi abbiamo udito che per timore delle persecuzioni, dissimulate a tal punto la vostra fede, e la nascondete, che comunicate pure con gli infedeli e andate alle loro abominevoli messe, nelle quali avete imparato a bestemmiare la morte e la passione di Cristo" (Prima risposta di Ecolampadio ai valdesi, 1530, 53).

Per i valdesi si apriva, dunque, l'esigenza di una riflessione religiosa profonda sul futuro delle proprie comunità, di una revisione delle loro concezioni dottrinali e di una professione pubblica della loro fede. Dal 12 al 18 settembre 1532 si tenne a Chanforan, in Val d'Angrogna, un Sinodo generale in cui intervennero delegati da tutte le comunità valdesi franco-italiane, comprese quelle di Puglia e Calabria, e nel quale si registrò una consistente partecipazione di fedeli, anche per via della rilevanza delle decisioni che avrebbe adottato l'assemblea, come testimonia lo stesso incipit della Dichiarazione del Sinodo: "Le propossicione che sono state disputate en angrogna lanno del segnor 1532. Et adi 12. de setembro. Enpresencia de tuti li ministri et ecian dio del populo" (Dichiarazione del sinodo di Chanforan, 1532, 139).

Il Sinodo di Chanforan ebbe un'importanza fondamentale per la storia del valdismo, traghettandolo dal pietismo medievale primigenio all'incontro con la "modernità" rappresentata dalla Riforma: determinò la trasformazione di un movimento ereticale clandestino in una chiesa strutturata e inserita nell'ampio 
movimento di rinnovamento religioso che agitava l'Europa. Di tale cambiamento abbiamo un riscontro significativo nella Dichiarazione di fede approvata a Chanforan (Foresta, 2011; Neuser, 1992; Gonnet, 1984; Subilia, 1982).

La Dichiarazione di Chanforan consta di 24 articoli, di cui due mancanti, il terzo e il quarto ${ }^{3}$, ciascuno giustificato con citazioni tratte dalle Sacre Scritture. La sua struttura risente delle esigenze di revisione delle pratiche tradizionali del valdismo rispetto al nuovo indirizzo: "la dottrina e la pietà valdese medievale sono interpretate secondo la fede della Riforma o del tutto respinte" (Vinay, 1974, 111).

Il documento di fede, dunque, non pose in primo piano i temi centrali della dottrina dei riformatori; la stessa giustificazione per fede non compare esplicitamente nella dichiarazione sinodale; ciò si spiega con la volontà del Sinodo di occuparsi innanzitutto dei temi controversi per i valdesi - quelle pratiche, a cominciare dalla scelta della povertà e della predicazione itinerante, che contraddistinguevano il movimento e alle quali si sarebbe dovuto rinunciare tralasciando le dottrine condivise": "l'ordine [degli articoli] è dunque determinato dalle questioni che stanno maggiormente a cuore ai Valdesi" (Vinay, 1974, 111).

La maggioranza delle propossicione è dedicata all'organizzazione e alla disciplina ecclesiastica, per cui la Dichiarazione appare più simile a un regolamento che a una confessione di fede, difatti i primi articoli riguardano il giuramento, le opere, la confessione auricolare, il riposo domenicale, il culto, l'imposizione delle mani; d'altronde il compito che il Sinodo si era dato era quello di edificare una chiesa regolarmente costituita, sul modello di quelle riformate (Subilia, 1982, 69-75).

Sul contenuto degli articoli di fede espressi a Chanforan ebbero un peso notevole le risposte rese per iscritto da Ecolampadio e Bucero ai quesiti posti dai valdesi e un ruolo di assoluto rilievo fu svolto da Guglielmo Farel, che partecipò personalmente ai lavori, esercitando un'influenza determinante sulle risoluzioni adottate dall'assemblea. Sicuramente l'influsso di Farel si può cogliere nella sottolineatura della dottrina della predestinazione, del "servo arbitrio" e della salvezza, nell'enunciazione degli articoli 19, 20 e 21 (Molnar, 1989, 223-24): “C. 19. Tuti quelli que sono stati et serano salvati sono preelleti avanti la costitutione del mondo. C. 20. Quelli que sono salvati non posseno

\footnotetext{
${ }^{3}$ Nell'originale della Dichiarazione la numerazione degli articoli passa direttamente dal 2 al 5.

${ }^{4}$ L'assenza di un riferimento esplicito alla dottrina della giustificazione per fede rimane comunque sorprendente, anche alla luce delle conversazioni sul tema dei barba Morel e Masson con i riformatori (Vinay, 1973).
} 
essere non salvati. [...] [C. 21.] ${ }^{5}$ Quicunque statuise elibero arbitrio denegua intuto la predestinatione et la gratia de dio" (Dichiarazione del sinodo di Chanforan, 1532, 142).

Una variazione considerevole rispetto al passato riguarda la materia sacramentale, con l'abbandono dei legami con la dogmatica cattolica riguardo ai sette sacramenti e alla dottrina della transustanziazione. Vennero adottate concezioni tipicamente riformate, in particolare sulla Santa Cena prevalse l'interpretazione che ne aveva dato Zwingli, mediata dai suggerimenti di Ecolampadio e Farel: "[C. 24. ${ }^{6}$ Circa la materia de li sacramenti he stato concluso per la scriptura che noy non havemo sinon doy segni sacramentali che Christo ne ha lasati luno he il baptismo laltro sie la heucarestia laquale noy usemo en demostramento la perseveracion nostra nela fede la quale havemo promesso nelo baptismo essendo filholi Et ancora alamemora dequello grande beneficio che Jesu Cristo ha facto anoy morendo per la redentione nostra elevando noy del suo pretioso sangue" (Dichiarazione del sinodo di Chanforan, 1532, 142).

Con il Sinodo di Chanforan, i valdesi abbandonarono le pratiche che avevano caratterizzato il movimento per oltre tre secoli: la rinuncia alla predicazione itinerante, il tratto più distintivo già dai tempi di Valdo, cambiò per sempre la fisionomia del valdismo. Si trattò, comunque, di una scelta che rispondeva alla necessità di superare una pratica divenuta anacronistica, dal momento che le comunità valdesi erano ormai insediate in un'area geografica ristretta (Molnar 1989, 220-24): "C. 22. Li menistri dela parolla de dio non si debeno mutare de loco jnloco sinon que sia agrande utilita dela chiesa" (Dichiarazione del sinodo di Chanforan, 1532, 142).

Insieme al ministero itinerante vennero abolite numerose pratiche tradizionali: la confessione auricolare (art. 5): "C. 5. La conffessione aurculare non he comandata da dio" (Dichiarazione del sinodo di Chanforan, 1532, 139); l'imposizione delle mani (art. 9), con la quale si ordinavano i barba: "C. 9. La inposicione de le mane non he necessaria" (Dichiarazione del sinodo di Chanforan, 1532, 140); la condizione di povertà (art. 23), elemento caratterizzante del messaggio di Valdo: "C. 23. Havere li menistri per nutrire la sua familha qualque cosa jnparticulare non he contra la communione apostolica" (Dichiarazione del sinodo di Chanforan, 1532, 142).

Gli articoli dal 13 al 16 si occupano dei temi del matrimonio e dell'abolizione del celibato per i ministri del culto: "C. 13. El matremonio non he proibito ha alcuno de quallunque stato ho ordine che sia. C. 14. Quicunque proibisse el matrimonio a quelli que el volleno ensegnano doctrina diabolica.

\footnotetext{
5 Nel testo originale manca la numerazione dell'articolo 21.

${ }^{6}$ Anche questo articolo è privo di numerazione.
} 
C. 15. Ordinare stato hovero ordine de verginita he doctrina diabolica. C. 16. Coluy che non ha el dono de continencia he obligato al matrimonio" (Dichiarazione del sinodo di Chanforan, 1532, 141).

L'eco del confronto dei valdesi con Ecolampadio e Bucero sul tema dell'usura $^{7}$, ritorna nelle formulazioni degli articoli 17 e 18, che riprendono le argomentazioni delle risposte dei due riformatori sulla legittimità del prestito a interesse, anticipatrici di quelle posizioni che successivamente esprimerà Giovanni Calvino (McGrath, 2009, passim): "C. 17. Non tuta usura he proibita de dio. Questo è chiaro perche dio non proibisse sinon lusura che agrava el proximo como contiene la lege non fare ha altro quello che non voresti che fosse facto acte. C. 18. Le parolle che sono en sancto luca dantes etc. non se intende de usura" (Dichiarazione del sinodo di Chanforan, 1532, 141-142).

La rilettura, in senso riformato, dell'epistola di San Paolo ai Romani trova accoglimento nell'articolo 11; qui non solo cadono le secolari riserve dei valdesi nei confronti dell'autorità civile, ma viene dichiarato legittimo l'esercizio delle funzioni politiche da parte dei cristiani: "C. 11. El christiano po exercitare el magistrato sopra li christiani de li quenti. La propossicione he clara como havemo en s. paulo Rom. 13. Corint. 6 c. e ensancto pietro en la soa prima epistolla" (Dichiarazione del sinodo di Chanforan, 1532, 141).

Riconosciuta l'obbedienza al potere civile, anche il giuramento diviene lecito qualora venga richiesto dall'autorità (art. 1): "C. 1. El christiano po iurare licitament" (Dichiarazione del sinodo di Chanforan, 1532, 139).

La Dichiarazione di Chanforan, sebbene influenzata dalle particolari circostanze fin qui riferite, si inquadra anch'essa nel clima della confessionalizzazione, particolarmente in ragione dell'esigenza di precisare una fisionomia dottrinale della propria fede nei confronti della chiesa di Roma e di rispondere efficacemente al tema dell'organizzazione e della disciplina ecclesiastica.

Queste importanti decisioni furono assunte dal Sinodo di Chanforan con la consapevolezza della portata delle trasformazioni che esse avrebbero comportato per il movimento valdese. I delegati, tuttavia, non furono unanimi nel recepire le innovazioni religiose della Riforma, anzi si delinearono tre tendenze: una raccoglieva gli "entusiasti” della svolta riformatrice; un'altra, più

7 "È proibita ogni usura che opprime il prossimo; ma dove qualcuno fa uso del guadagno ottenuto dall'altrui lavoro, cioè riceve l'uso di un capitale dato in prestito, deve certamente dare qualcosa al suo prossimo del guadagno ricavato con il lavoro di lui. Così avverrà che ricevere denaro da denaro non sia sempre un'usura proibita" (Risposta di Martin Bucero ai fratelli Valdesi in Francia, 1530, 109); "noi non siamo così severi da chiamare usurai tutti quelli che fanno prestiti e poi prendono qualche interesse" (Prima risposta di Ecolampadio ai valdesi, 1530, 57). 
cauta, era favorevole all'adesione alle chiese riformate, mantenendo, però, alcune caratteristiche proprie del valdismo; la terza, conservatrice, respingeva le innovazioni, in nome di un tradizionalismo da preservare. La mancata unanimità è confermata anche dall'esortazione finale della Dichiarazione, che sottolinea la necessità di mantenere l'unità del movimento: "Ve pergiuriamo nele viscere dela carita che da poy che noy saremo partiti de jnsieme que non ne siamo discordanti nelo jnsegnare tanto nele desuso dicte conclusione coma nela jnterpretatione dela scriptura et cosi como uno medesimo sperito la composta faciamo che sia jnterpretata per questo medessimo sperito" (Dichiarazione del sinodo di Chanforan, 1532, 143).

La componente conservatrice, insoddisfatta dell'esito del Sinodo, ritenne opportuno interpellare i Fratelli Boemi - movimento nato dalla fazione più radicale dell'hussitismo in stretta relazione con le pratiche e le dottrine del valdismo boemo e con il quale esistevano antiche e fraterne relazioni (Molnar, 1989, 159-96; Molnar, 1986, 5-45) - inviando due barba, Daniele di Valenza e Giovanni da Molines, per informarli degli eventi accaduti nella comunità valdese franco-italiana. Delle "dolorose impressioni" che i Fratelli ricavarono dai racconti dei due barba, ne abbiamo testimonianza diretta nella lettera che essi scrissero ai valdesi: "Sono giunti da noi in Boemia i vostri fratelli, Daniele e Giovanni [...] ci hanno fatto conoscere fino a qual punto certi Svizzeri, che non sappiamo se giuochino con le Sacre Scritture e la dottrina cristiana o le corrompano, si siano intromessi fra voi; vi abbiano turbati con varie questioni riguardanti la dottrina della salvezza, sì che hanno provocato un doloroso scisma anche tra voi, che per tanti secoli siete stati uniti, per rendervi straordinariamente inquieti. [...] Per questo fatto non abbiamo potuto non provare grande dolore" (I Fratelli di Boemia e di Moravia ai Valdesi, 1533, 145).

La critica mossa dai Fratelli Boemi, sulla base di quanto riportato dai barba Daniele e Giovanni, riguardava fondamentalmente la leggerezza e la precipitosità delle scelte compiute, "non piccolo stupore ci coglie, che voi tanto presto vi siate lasciati ingannare" (I Fratelli di Boemia e di Moravia ai Valdesi, 1533, 147).

Al ritorno dei due barba nelle Valli, la lettera e le argomentazioni provenienti dalla Boemia furono ampiamente oggetto di discussione nelle comunità valdesi: il Sinodo di Prali, che si svolse nell'agosto del 1533, confermò, comunque, le decisioni di Chanforan, a dimostrazione della consapevolezza con cui il movimento valdese aveva intrapreso il nuovo cammino (Molnar, 1989, 232-35).

Il processo di adesione dei valdesi alla Riforma, pertanto, non fu limitato al solo Sinodo di Chanforan, ma lo si può collocare in un arco cronologico che iniziò a Laus nel 1526 e si protrasse fino al Sinodo di Prali del 1533, dove la nuova fede evangelica professata a Chanforan venne confermata. I primi effetti delle decisioni sinodali furono l'istituzione del culto pubblico - con il conse- 
guente abbandono delle pratiche nicodemitiche - e di un ministero ecclesiastico stabile. Nei decenni successivi si perfezionarono l'organizzazione delle comunità in parrocchie, la costruzione dei luoghi di culto, la tenuta dei registri, la preparazione dei pastori attraverso il loro invio nelle accademie di Ginevra, Losanna e Basilea e l'adozione del sistema presbiteriano-sinodale di matrice calvinista (Armand Hugon, 1989, 13-19; Gonnet, 1981).

\section{LA CONFESSIONE VALDESE DEL 1655}

All'affermazione e al consolidamento della Riforma contribuì in modo decisivo il clima di agitazioni sociali e di conflitti politici dell'Europa del XVI secolo, a partire dallo scontro che opponeva i principi territoriali tedeschi all'imperatore Carlo V. La Riforma poté contare sull'appoggio di autorità politiche che, quando le circostanze lo resero possibile - dopo la pace di Augusta del 1555 con l'affermazione del principio cuius regio eius religio - la imposero ai loro sudditi. I valdesi, al contrario, si trovarono costantemente costretti a fronteggiare l'ostilità del potere temporale, a subire dure campagne persecutorie dettate dalla politica di confessionalizzazione romana e messe in atto dai sovrani francesi e dai Savoia.

Nella primavera del 1655 - "la primavera di sangue" (Armand Hugon, 1989, 73) - con il pretesto della violazione dell' accordo di Cavour del 1561, che limitava l'ambito della predicazione e dell'esercizio del culto valdese alle sole valli alpine, lo Stato Sabaudo avviò una nuova campagna di feroci repressioni, con uccisioni, saccheggi, violenze, incluso il rapimento di fanciulli affinché "venissero educati alla fede cattolica" (Campi, 2001, 88). Tra i diversi strumenti messi in atto dai valdesi per richiamare l'attenzione su quanto accadeva nelle Valli, vi fu la pubblicazione e la diffusione di testi che denunciavano i massacri perpetrati dalle truppe piemontesi. Questi opuscoli, che circolarono in gran parte d'Europa, servirono a sensibilizzare il mondo protestante e ad avviare le iniziative diplomatiche inglese e svizzera volte a frenare le violenze sabaude (McComish, 1981; Vola, 1981; Balmas, Menascé, 1981; Contino, 1953).

Uno di questi opuscoli, la Relation veritable de ce qui s'est passé dans les persecutions \& massacres faits cette année, aux Eglises Reformées de Piémont. Avec la refutation des calomnies dont les Adversaires de la vérité taschent de les noircir. MDCLV, nella sua terza edizione, conteneva una confessione di fede in lingua francese, la Brieve Confession de foy des Eglises Reformées de Piémont, probabilmente approvata da un Sinodo svoltosi ad Angrogna nel 1655.

Se la paternità dell'opuscolo è attribuibile ad Antoine Léger (De Lange, 1997; Tron, 1998, 5), - esule valdese, pastore e professore dell'Accademia di 
Ginevra - riguardo alla confessione di fede, a oggi, gli storici non concordano su un unico autore: Revel e Vinay ritengono che l'autore della confessione sia Jean Léger, nipote di Antoine (Revel, 1878; Vinay, 1975, 30), Tron individua invece il possibile autore nel collega di Antoine Léger, Théodore Tronchin (Tron, 1998, 9-10). È certo, comunque, che Antoine Léger ne tradusse il testo in italiano all'interno di un volume scritto nel 1661 e pubblicato postumo l'anno successivo, l'Apologia delle Chiese riformate del Piemonte, circa la loro confessione di fede e la continua successione di esse tanto nei natii del paese quanto nei Valdesi contro le gavillationi e calunnie del Priore Marco Aurelio Rorengo di Lucerna: si trattava di una risposta alla polemica sollevata dal priore di Luserna, Marco Aurelio Rorengo, che nel 1658 aveva pubblicato un libello - Essame intorno alla nuova breve confessione di fede delle Chiese Riformate di Piemonte in cui si fa conoscer alli professori d'esse, distrutte con la novità, la pretesa antica confessione - con il quale confutava la nuova confessio valdese. Questa controversia teologica si collocava pienamente nel clima di contrapposizione religiosa dell'epoca: Rorengo e Léger erano due rappresentanti di identità confessionali ben definite, cattolico-romana post-tridentina, da un lato, e protestante-riformata, nella sua definizione del Sinodo di Dordrecht, dall'altro (Gajewski, 2008).

La confessione di fede del 1655 (CF/1655), elaborata in un momento drammatico della storia del movimento valdese, segue l'impostazione e il contenuto, in versione ridotta, della Confessio Gallicana del 1559, documento di fede delle chiese riformate di Francia (Campi, 1991, 179-83). La CF/1655, composta da 33 articoli, invece dei 40 della Gallicana, è una confessione che presenta, in forma succinta, le principali dottrine della chiesa riformata valdese: se la Dichiarazione del Sinodo di Chanforan è una testimonianza del passaggio del valdismo medievale alla Riforma, la $\mathrm{CF} / 1655$ presenta formulazioni mature, proprie di una chiesa ormai dotata di una precisa identità confessionale.

$\mathrm{La} \mathrm{CF} / 1655$ si apre con un'introduzione che spiega le ragioni della sua formulazione: non solo denunciare gli abusi, le persecuzioni e le spoliazioni subite, ma soprattutto respingere le calonnie cui la fede valdese era sottoposta, dimostrando la correttezza delle dottrine e l'adesione alla Parola di Dio: "Avendo inteso che i nostri Avversarii, non contentandosi di haverci crudelmente perseguitati e spogliati de' nostri beni, per renderci vie più odiosi, vanno ancora seminando de' falsi rumori tendenti non solo a macchiare le nostre persone, ma principalmente a denigrare con calonnie infami la santa e salutare dottrina, la quale professiamo; Noi siamo obligati per chiarire lo spirito di quelli che potrebbono essere preoccupati di tali sinistri pensieri, di fare una breve dichiaratione della nostra fede, quale l'habbiamo per l'addietro avuta, e la teniamo ancora hoggidì, conforme alla Parola de Dio, acciocché ognuno vegga 
la falsità di quelle calonnie e con quanta ingiustitia siamo odiati e perseguitati per una sì innocente dottrina" (CF,1655, 7).

La confessione è strutturata in articoli, ciascuno dei quali è corredato da "prove" che giustificano il testo sulla base di passi biblici, quasi sempre spiegati e attualizzati: "Articolo 1 - (Trinità) Che vi è un solo Iddio, il quale è una Essenza spirituale, eterna, infinita, del tutto savia, misericordiosa, giusta, in somma del tutto perfetta; e che vi sono tre Persone in quella sola e semplice essenza, il Padre, il Figliuolo e lo Spirito Santo. PROVE Deut. 4,39 - Isa. 42,8; 45,5. - Non è dunque licito appoggiar la fiducia e speranza nostra nelle creature, ne invocar li Santi, ne adorar le imagini, o la croce, atteso che queste cose non sono Dio. - Esodo 20,2 - Isa. 40,18 e 25. - Contra questo la Chiesa Romana rappresenta Iddio in habito Papale, benche S. Paolo lo habbia condannato [...] Il mistero della Santa e adorabile Trinità c'è insegnato nella Santa Scrittura, come si vede ne' luoghi seguenti, Matt. 28,19 - II Cor. 13,13 - I Giov. 5,7” (CF,1655, 7-8).

I caratteri più marcatamente "protestanti" della $\mathrm{CF} / 1655$ emergono in numerosi articoli; per esempio nell'articolo 28, dove si ribadisce il riconoscimento di due soli sacramenti, il Battesimo e la Santa Cena: "Che Iddio non ci ammaestra solo colla sua parola, ma che di più egli ha ordinati de' Sacramenti per congiungergli ad essa, come mezzi per unirci a Christo e partecipar alli suoi benefici, e che non ve ne sono più di due comuni a tutte le membra della Chiesa, sotto "l Nuovo Testamento, ciò è il Battesimo e la Santa Cena" $(\mathrm{CF}, 1655,20)$; l'articolo 16 riafferma la giustificazione in virtù del solo merito di Cristo: "Che il Signor Jesu havendoci pienamente riconciliati a Dio con il sangue della sua croce, in virtù del suo solo merito e non delle nostre opere, noi siamo assolti e giustificati nel suo cospetto" (CF,1655, 14).

La dottrina della predestinazione in senso supralapsario, di indubbia influenza riformata ${ }^{8}$ viene espressa negli articoli 11 e 25: "Articolo 11 - (Elezione) Che Iddio cava da quella corruttione e condannatione le persone ch'egli ha elette dinanzi la fondatione del mondo [...] Articolo 25 - (Definizione della Chiesa) Che quella Chiesa è la compagnia de' fedeli, i quali essendo stati da

${ }^{8}$ La concezione supralapsaria della predestinazione (antecedente la caduta di Adamo), uno dei caposaldi dell'ortodossia riformata, si impose definitivamente con il Sinodo di Dordrecht del 1618, nel quale le teorie arminiane (da Giacomo Arminio), infralapsarie, vennero ufficialmente condannate. Arminio, a differenza dei calvinisti ortodossi -detti anche gomaristi in quanto seguaci delle teorie di Francesco Gomar-, riteneva che la predestinazione fosse collegata alla "perseveranza" nella fede, ipotizzando un'accettazione umana della grazia. Lo scontro ben presto investì anche il campo della politica, in particolare nei Paesi Bassi, con i gomaristi sostenitori delle pretese monarchiche degli Orange e della guerra contro la Spagna, e gli arminiani fautori della repubblica e di una politica estera non-belligerante (Fiume, 2015; Campi, 1991, 272-77). 
Dio eletti avanti la fondatione del mondo, e chiamati d'una santa vocatione, vengono ad unirsi per seguitare la Parola di Dio" (CF,1655, 12 e 18).

Se da un lato la $\mathrm{CF} / 1655$ accoglie con chiarezza i principi e i fondamenti propri di una Chiesa riformata, dall'altro tende ad affermare senza ambagi, in diversi articoli, la distanza nei confronti della dottrina cattolica: la ripetizione del sacrificio di Cristo nella messa (art.15): "il vero sacrificio di Christo essendo fatto una sola volta, non conviene reiterarlo, come si pretende nella messa" (CF,1655, 14); il rifiuto del culto dei santi (art. 23): "Che quelli che possegono la vita eterna in conseguenza della fede e delle buone opere loro deono esser considerati come santi e glorificati, lodati per le loro virtù, imitati in tutte le belle attioni della loro vita, ma non adorati ne invocati, poi che non si deve pregar se non un solo Iddio per Jesu Christo" (CF,1655, 17); la negazione del valore salvifico delle opere (art. 22): "li fedeli non possono pretender la vita eterna come salario meritato per le loro buone opere, anzi che la conseguiscono come un dono gratuito di Dio" (CF,1655, 17); Cristo come unico capo della chiesa, in opposizione al pontefice (art. 24): "ch'ella non ha se non un solo Capo e fondamento, cioè Jesu Christo [...] come dunque non ha vergogna il Papa di vantarsi d'esser capo e sposo della Chiesa?" (CF,1655, 18); il rifiuto della dottrina della transustanziazione (art. 30): "la carne e '1 sangue di Christo non si ricevono corporalmente nel Santo Sacramento [...] ma spiritualmente per fede" (CF,1655, 22).

Sono ben evidenti anche nella $\mathrm{CF} / 1655$ le influenze dell'ormai declinante processo di confessionalizzazione - a partire dalla fine della guerra dei Trent'anni il peso della confessionalizzazione decrebbe in tutto il continente europeo (Lotz-Heumann, 2001) - in ordine alla definizione della propria dottrina, in antitesi soprattutto a quella cattolico-romana, ma anche delle forme del culto e dell'organizzazione ecclesiastica. La Confessione del 1655, inoltre, assume un'importanza particolare proprio perché prodotta all'indomani della sanguinosa aggressione controriformistica delle "Pasque piemontesi", cui il movimento valdese seppe valorosamente resistere (Armand Hugon, 1989, 73-102).

Nella conclusione della CF/1655 i valdesi fanno appello alla comune fede evangelica che, pur nelle differenze tra luterani e riformati, caratterizza il mondo protestante. Vengono menzionate le chiese riformate nazionali, alle quali si richiede sostegno e protezione; la solidarietà internazionale risultò provvidenziale per la stessa sopravvivenza del movimento valdese: "consentiamo nella sana dottrina con tutte le Chiese Riformate di Francia, della Gran Bretagna, de' Paesi Bassi, Alamagna, Svizzeri, Boemia, Polonia, Ungaria et altre, com'ella è rappresentata nella loro Confessione d'Augusta [...] Preghiamo umilmente tutte le Chiese Evangeliche e Protestanti di tenerci nonostante la nostra povertà e bassezza per vere membra del corpo mistico di Jesu Christo che sofferiscono pe 'l 
suo nome, e di continuarci l'aiuto delle loro preghiere verso Iddio, e tutti gli altri effetti della loro carità, come già gli habbiamo copiosamente provati; Onde le ringratiamo con tutta l'humiltà possibile" $(\mathrm{CF}, 1655,25)$.

La CF/1655, tradotta in italiano da Léger e pubblicata nel 1662 - per questo, a volte, impropriamente chiamata "Confessione del 1662" (Tron, 1998, 14) - venne confermata da due successivi sinodi, nel 1839 e nel 1855, e da quel momento divenne la confessione di fede ufficiale della chiesa Valdese. Nel Sinodo del 1894 venne integrata da un Atto dichiarativo (Atto Dichiarativo del Sinodo del 1894, 2009), che ne reinterpretava alcuni articoli. Ancora oggi, i pastori valdesi devono sottoscriverla prima di essere consacrati.

\section{CONCLUSIONI}

Le confessioni di fede prese in esame in questo studio vennero elaborate in occasione di due passaggi cruciali per il movimento valdese: il Sinodo di Chanforan, che avrà un'importanza cardinale per gli sviluppi successivi del valdismo, e le "Pasque Piemontesi", uno dei momenti più problematici e tormentati nella storia delle comunità evangeliche delle valli alpine occidentali.

Si tratta di documenti di fede dal profilo, dalle caratteristiche e dalla struttura molto differenti. La Dichiarazione di Chanforan, rappresenta, dal punto di vista formale e dottrinale, un unicum, in quanto segnala nitidamente la trasformazione del valdismo da movimento pauperistico in Chiesa riformata; gli articoli di questa confessione, che come abbiamo visto nelle pagine precedenti presentano talvolta i tratti di un regolamento, testimoniano l'abbandono del pietismo medievale e di quell'insieme di pratiche che fino a quel momento avevano caratterizzato il movimento; al contempo annunciano l'adozione dei principi, tanto dottrinali come organizzativi, della Riforma.

La CF/1655, redatta oltre un secolo dopo Chanforan, presenta, invece, le caratteristiche tipiche dei documenti di fede dell'epoca confessionale: da un lato si espongono una serie di articoli tesi a definire dogmaticamente la Chiesa - in senso manifestamente riformato -, dall'altra si ricusano le dottrine avverse, cattolico-romane, rifiuto qui ancor più enfatizzato dalle particolari vicende politiche e religiose all'origine del documento. $\mathrm{La} \mathrm{CF} / 1665$, redatta sul modello della confessione di fede delle Chiese riformate di Francia, attesta l'avvenuto e pieno rinnovamento del valdismo all'interno del più ampio e generale movimento protestante come confermato, tra le altre cose, dall'accorato appello alle chiese riformate e luterane con il quale si chiude la confessione di fede. 


\section{FONTI}

“Atto Dichiarativo del Sinodo del 1894", in: Raccolta delle discipline vigenti nell'ordinamento valdese (2009), Torino, Claudiana, 27-30.

"I barba Georges Morel e Pierre Masson a Ecolampadio", in: Vinay, V. (Ed.), Le confessioni di fede dei valdesi riformati. Con documenti del dialogo fra «prima»e «seconda» Riforma (1975), Torino, Claudiana, 37-51.

"Confessione di fede valdese (1655)", in: Raccolta delle discipline vigenti nell'ordinamento valdese (2009), Torino, Claudiana, 3-26.

"Dichiarazione del sinodo di Chanforan (1532)", in: Vinay, V. (Ed.), Le confessioni di fede dei valdesi riformati. Con documenti del dialogo fra «prima»e «seconda» Riforma (1975), Torino, Claudiana, 139-143.

"I Fratelli di Boemia e di Moravia ai Valdesi", in: Vinay, V. (Ed.), Le confessioni di fede dei valdesi riformati. Con documenti del dialogo fra «prima» e «seconda» Riforma (1975), Torino, Claudiana, 145-151.

"Prima risposta di Ecolampadio ai valdesi", in: Vinay, V. (Ed.), Le confessioni di fede dei valdesi riformati. Con documenti del dialogo fra «prima» e «seconda» Riforma (1975), Torino, Claudiana, 53-63.

"Risposta di Martin Bucero ai fratelli Valdesi in Francia", in: Vinay, V. (Ed.), Le confessioni di fede dei valdesi riformati. Con documenti del dialogo fra "prima»e «seconda» Riforma (1975), Torino, Claudiana, 75-117.

\section{BIBLIOGRAFIA}

ARMAND HUGON, A. (1989): Storia dei valdesi 2. Dal sinodo di Chanforan all'emancipazione, Torino, Claudiana, $327 \mathrm{p}$.

AUDISIO, G. (2007): Preachers by night. The Waldensian barbes (15th-16th centuries), Leiden-Boston, Brill, $258 \mathrm{p}$.

BALMAS, E., MENASCÉ, E. (1981): "L'opinione pubblica inglese e le 'Pasque Piemontesi': nuovi documenti”, Bollettino della Società di Studi Valdesi, 150, 3-26.

CAMPI, E. (1991): Protestantesimo nei secoli. Fonti e documenti, Cinquecento e Seicento, Torino, Claudiana, $474 \mathrm{p}$.

CAMPI, E. (2001): "Nascita e sviluppi del protestantesimo (secoli XVI-XVIII)", in: Filoramo, G., Menozzi, D., Storia del Cristianesimo. L'età Moderna, Roma-Bari, Editori Laterza, 3-150.

CEGNA, R. (1981): "Il valdismo medievale come religione penitenziale", Bollettino della Società di Studi Valdesi, 150, 37-50.

COMBA, E. (1932): "I valdesi prima del sinodo di Cianforan", Bollettino della Società di Storia Valdese, 54, 7-33.

CONTINO, T. (1953): "L'intervento diplomatico inglese a favore dei valdesi in occasione delle pasque piemontesi del 1655", Bollettino della Società di Studi Valdesi, 94, 35-43. 
FIUME, E. (2015): Il Sinodo di Dordrecht (1618-1619). Predestinazione e calvinismo. Torino, Claudiana, $135 \mathrm{p}$.

FORESTA, P. (2011): "Da barba a pastori: il concilium generale di Chanforan (1532)", Cristianesimo nella storia: ricerche storiche esegetiche teologiche, 32, 733-53.

GAJEWSKI, P. (2008): “Uso dell'argomentazione biblica in funzione polemica in campo cattolico e riformato sull'esempio del priore Marco Aurelio Rorengo e del pastore Antoine Léger", Bollettino della Società di Studi Valdesi, 202, 245-64.

GONNET, G. (1972): "La donazione di Costantino presso gli eretici medioevali", Bollettino della Società di Studi Valdesi, 132, 17-30.

GONNET, G. (1981): "Mérindol: fine del valdismo?", Bollettino della Società di Studi Valdesi, 150, 27-36.

GONNET, G. (1984): “Chanforan e la storiografia valdese”, Bollettino della Società di Studi Valdesi, 154, 3-24.

LANGE, A. De. (1997): “Antoine Léger (1596-1661), un 'internazionalista' calvinista del Seicento”, Bollettino della Società di Studi Valdesi, 181, 203-33.

LOTZ-HEUMANN, U. (2001): “The concept of ‘confessionalization': a historiographical paradigm in dispute", Memoria y Civilización, 4, 93-114.

MCCOMISH, W. (1981): "Reazioni inglesi alla 'primavera di sangue' valdese del 1655", Bollettino della Società di Studi Valdesi, 149, 3-10.

MCGRATH, A.E. (2009): Giovanni Calvino. Il riformatore e la sua influenza sulla cultura occidentale, Torino, Claudiana, $344 \mathrm{p}$.

MERLO, G.G. (1984): Valdesi e valdismi medievali, Torino, Claudiana, 158 p.

MERLO, G.G. (2010): Valdo. L'eretico di Lione, Torino, Claudiana, 120 p.

MOLNAR, A. (1976): A challenge to constantinianism. The Waldensian theology in Middle Ages, Genève, WSCF, 74 p.

MOLNAR, A. (1986): I Taboriti: avanguardia della rivoluzione hussita (sec. XV), Torino, Claudiana, $200 \mathrm{p}$.

MOLNAR, A. (1989): Storia dei valdesi 1. Dalle origini all'adesione alla riforma, Torino, Claudiana, $372 \mathrm{p}$.

NEUSER, W. (1992): "Das waldenserbekenntnis von Chanforan 1532: ein reformiertes bekenntnis?", HTS Teologiese Studies/Theological Studies, XLVIII (3-4), 861-69.

POLASTRO, M. (1974): "Ecclesiologia valdese”, Bollettino della Società di Studi Valdesi, 136, 117-32.

REINHARD, W. (1977): "Gegenreformation als modernisierung? Prolegomena zu einer theorie des konfessionellen zeitalters", Archiv Für Reformationsgeschichte, LXVIII, 226-51.

REVEL, A. (1878): "I simboli della chiesa valdese", Rivista Cristiana, 6, 7-21.

SCHILLING, H. (1981): Konfessionskonflikt und staatsbildung, Gütersloh, 443 p.

SUBILIA, V. (1982): "Chanforan 1532 o la presenza protestante in Italia”, Protestantesimo, XXXII, 65-94.

TRON, D. (1998): “Indagini sulla confessione di fede valdese del 1655. Una messa a punto su cronologia e luoghi di stampa delle varie versioni (una tra le quali sconosciuta sin qui); con ipotesi sull'ambiente di elaborazione del testo, ed un'edizione critica bilingue del medesimo", Bollettino della Società di Studi Valdesi, 183, 3-41. 
VINAY, V. (1973): "Barba Morel e Bucero sulla giustificazione per fede", Bollettino della Società di Studi Valdesi, 133, 29-36.

VINAY, V. (1974): "Il breve dialogo fra prima e seconda riforma 1530-1533", Bollettino della Società di studi Valdesi, 136, 99-116.

VINAY, V. (1975): Le confessioni di fede dei valdesi riformati. Con documenti del dialogo fra «prima»e «seconda» riforma, Torino, Claudiana, 209 p.

VOLA, G. (1981): "Cromwell e i valdesi, una vicenda non del tutto chiarita", Bollettino della Società di Studi Valdesi, 149, 11-37. 
